\title{
Improved inclusion-exclusion identities via closure operators
}

\author{
Klaus Dohmen \\ Department of Computer Science, Humboldt-University Berlin, Unter den Linden 6, D-10099 Berlin, Germany \\ E-mail: dohmen@informatik.hu-berlin.de
}

received March 24, 1999, revised September 6, 1999, accepted April 15, 2000.

Let $\left(A_{v}\right)_{v \in V}$ be a finite family of sets. We establish an improved inclusion-exclusion identity for each closure operator on the power set of $V$ having the unique base property. The result generalizes three improvements of the inclusion-exclusion principle as well as Whitney's broken circuit theorem on the chromatic polynomial of a graph.

Keywords: inclusion-exclusion, sieve formula, closure operator, convex geometry, broken circuit, reliability

\section{Introduction}

One of the most important tools in enumerative combinatorics and combinatorial probability theory is the principle of inclusion-exclusion, which is also known as the sieve formula or the formula of Poincaré or Sylvester. A detailed account of the principle of inclusion-exclusion (and the associated Bonferroni inequalities) is given in the textbook of Galambos and Simonelli [9].

For any finite family $\left(A_{v}\right)_{v \in V}$ of sets the principle of inclusion-exclusion states that

$$
\chi\left(\bigcup_{v \in V} A_{v}\right)=\sum_{\substack{J \subseteq V \\ J \neq \emptyset}}(-1)^{|J|-1} \chi\left(\bigcap_{j \in J} A_{j}\right),
$$

where for any set $A, \chi(A)$ denotes the indicator function of $A$, that is,

$$
\chi(A)(\omega)= \begin{cases}1 & \text { if } \omega \in A \\ 0 & \text { otherwise }\end{cases}
$$

There is no real restriction in using indicator functions rather than measures, since Eq. (1) can be integrated with respect to any measure (e.g., the counting measure) on the algebra generated by the family $\left(A_{v}\right)_{v \in V}$.

Since the sum on the right-hand side of Eq. (1) ranges over $2^{|V|}-1$ terms, it is natural to ask whether fewer terms would give the same result. Partial answers to this question are given by several authors, e.g., McKee [10,11], Naiman and Wynn [12,13], Narushima [14,15] and the present author [1-5]. The 1365-8050 @ 2000 Maison de l'Informatique et des Mathématiques Discrètes (MIMD), Paris, France 
problem naturally arises in several situations, e.g., when assessing the reliability of a network $[4,17]$ or when computing the volume of a union of balls or other geometric objects in Euclidean space [7,8,12,13].

In the proof of his famous inclusion-exclusion variant for semilattices, Narushima [15] uses a prominent result of Rota [16] on closure operators and Möbius functions. Although the significance of closure operators becomes obvious by Narushima's proof and Rota's work, no inclusion-exclusion variant has been established that contains a closure operator in its premises or hypothesis. In this paper, we establish such a variant and deduce three results from it, which for the first time appear in a common context.

\section{Improved inclusion-exclusion identities}

For any set $V$, let $\mathcal{P}(V)$ denote the set of non-empty subsets of $V$. A closure operator on $\mathcal{P}(V)$ is a mapping $X \mapsto X^{*}$ from $\mathcal{P}(V)$ into itself satisfying

$$
\begin{aligned}
\text { (i) } & X \subseteq X^{*} \\
\text { (ii) } & X \subseteq Y \Rightarrow X^{*} \subseteq Y^{*} \\
\text { (iii) } & X^{* *}=X^{*}
\end{aligned}
$$

for any $X, Y \in \mathcal{P}(V)$. A set $X \in \mathcal{P}(V)$ is closed if $X^{*}=X . X$ is perfectly closed if all non-empty subsets of $X$ are closed. A base of $X$ is a minimal non-empty subset $B$ of $X$ such that $B^{*}=X$. Evidently, if the underlying set is finite, then each closed set has a base. A closure operator is said to have the unique base property if none of its closed sets has more than one base.

Closure operators having the unique base property may be viewed as natural generalizations of the convex hull operator. Thus, there is a strong connection between our work and that of Edelman and Jamison [6] on convex geometries.

Proposition 1 Let $V$ be a finite set, $X \mapsto X^{*}$ a closure operator on $\mathcal{P}(V)$ and $J \in \mathcal{P}(V)$. Then, $J$ is a base of itself if and only if $J$ is perfectly closed.

Proof. If $J$ is perfectly closed then $J^{*}=J$ and if $K \subset J$ is non-empty then $K^{*}=K \subset J$, so $J$ is a base of itself. On the other hand, assume that $J$ is closed, but not perfectly closed, that is, $J^{*}=J$ and $K \subset J$ for some non-empty and non-closed set $K$. Then, pick $k \in K^{*} \backslash K$. So $k \in K^{*} \subseteq(J \backslash\{k\})^{*}$ and $J \backslash\{k\} \subseteq(J \backslash\{k\})^{*}$. Taking the union of these last two containments, $J \subseteq(J \backslash\{k\})^{*}$, so $(J \backslash\{k\})^{*}=J$ where $k \in J$, whence $J$ is not a base of itself.

Proposition 2 Let $\left(A_{v}\right)_{v \in V}$ be a family of sets and $X \mapsto X^{*}$ be a closure operator on $\mathcal{P}(V)$ such that for any non-empty and non-closed subset $X$ of $V$,

$$
\bigcap_{x \in X} A_{x} \subseteq \bigcup_{v \notin X} A_{v} .
$$

Then, for any non-empty subset $J$ of $V$,

$$
\bigcap_{j \in J} A_{j}=\bigcap_{j \in J^{*}} A_{j}
$$


Proof. Fix some $J \subseteq V, J \neq \emptyset$. There is nothing to prove if $\bigcap_{j \in J} A_{j}=\emptyset$. Otherwise choose $\omega \in \bigcap_{j \in J} A_{j}$ and show that $\omega \in \bigcap_{j \in J^{*}} A_{j}$. By the choice of $\omega, J \subseteq V_{\omega}$ where $V_{\omega}:=\left\{v \in V \mid \omega \in A_{v}\right\}$. By the definition of $V_{\omega}$ and (3), $V_{\omega}$ is closed and hence, $J^{*} \subseteq V_{\omega}$. Hence, $\omega \in \bigcap_{j \in J^{*}} A_{j}$ and the proposition is proved.

The following proposition from [6] generalizes and simplifies a Proposition of Narushima [15, p. 198].

Proposition 3 Let $V$ be a finite set and $X \mapsto X^{*}$ be a closure operator on $\mathcal{P}(V)$ having the unique base property. Then, for each closed set $J \in \mathcal{P}(V)$,

$$
\sum_{\substack{I \in \mathcal{P}(J) \\ I^{*}=J}}(-1)^{|I|}= \begin{cases}(-1)^{|J|} & \text { if } J \text { is perfectly closed } \\ 0 & \text { otherwise }\end{cases}
$$

Proof. Let $J_{0}$ be the unique base of $J$. Then, $I^{*}=J$ if and only if $J_{0} \subseteq I \subseteq J$. Hence,

$$
\sum_{\substack{I \in \mathcal{P}(J) \\ I^{*}=J}}(-1)^{|I|}= \begin{cases}(-1)^{|J|} & \text { if } J_{0}=J \\ 0 & \text { otherwise. }\end{cases}
$$

By Proposition $1, J_{0}=J$ if and only if $J$ is perfectly closed, whence the result.

Theorem 1 Let $\left(A_{v}\right)_{v \in V}$ be a finite family of sets and $X \mapsto X^{*}$ be a closure operator on $\mathcal{P}(V)$ satisfying the unique base property and

$$
\bigcap_{x \in X} A_{x} \subseteq \bigcup_{v \notin X} A_{v}
$$

for any non-empty and non-closed subset $X$ of $V$. Then,

$$
\chi\left(\bigcup_{v \in V} A_{v}\right)=\sum_{\substack{J \in \mathcal{P}(V) \\ J \text { perf. closed }}}(-1)^{|J|-1} \chi\left(\bigcap_{j \in J} A_{j}\right) .
$$

Proof. By the classical inclusion-exclusion principle,

$$
\chi\left(\bigcup_{v \in V} A_{v}\right)=\sum_{\substack{J \in \mathcal{P}(V) \\ J *=J}} \sum_{\substack{I \in \mathcal{P}(J) \\ I^{*}=J}}(-1)^{|I|-1} \chi\left(\bigcap_{i \in I} A_{i}\right) .
$$

From this, the requirements of the theorem and Proposition 2 we conclude that

$$
\chi\left(\bigcup_{v \in V} A_{v}\right)=\sum_{\substack{J \in \mathcal{P}(V) \\ J^{*}=J}} \sum_{\substack{I \in \mathcal{P}(J) \\ I^{*}=J}}(-1)^{|I|-1} \chi\left(\bigcap_{j \in J} A_{j}\right) .
$$

Now, by applying Proposition 3, the result follows.

Note that by setting $X^{*}:=X$ for any non-empty subset $X$ of $V$, Theorem 1 specializes to the classical inclusion-exclusion principle. 
As a first corollary we now deduce the semilattice sieve of Narushima [15]. Recall that a join-semilattice is a partially ordered set $V$ such that every two elements $x, y \in V$ have a least common upper bound, which we denote by $x \vee y$. A chain in $V$ is a subset $C$ of $V$ such that any two elements of $C$ are comparable.

Corollary 1 (Narushima) Let $\left(A_{v}\right)_{v \in V}$ be a finite family of sets, where $V$ is a join-semilattice such that $A_{x} \cap A_{y} \subseteq A_{x \vee y}$ for any $x, y \in V$. Then,

$$
\chi\left(\bigcup_{v \in V} A_{v}\right)=\sum_{\substack{J \in \mathcal{P}(V) \\ J \text { is a chain }}}(-1)^{|J|-1} \chi\left(\bigcap_{j \in J} A_{j}\right) .
$$

Proof. As in [6], let $X^{*}$ be the join-subsemilattice generated by $X$. Then, each closed set $X$ has a unique base, consisting of its join-irreducibles. (Recall that $x$ is join-irreducible if $x=a \vee b$ implies $x=a$ or $x=b$.) Clearly, $X$ is perfectly closed if and only if $X$ is a chain. Condition (7) holds since any non-closed set $X \neq \emptyset$ contains $x$ and $y$ such that $A_{x} \cap A_{y} \subseteq A_{x \vee y}$ and $x \vee y \notin X$. Theorem 1 now gives the result.

As a second corollary we deduce the tree sieve of Naiman and Wynn [13].

Corollary 2 (Naiman-Wynn) Let $\left(A_{v}\right)_{v \in V}$ be a finite family of sets, where the indices form the vertices of a tree $G=(V, E)$ such that $A_{x} \cap A_{y} \subseteq A_{z}$ for any $x, y \in V$ and any $z$ on the path from $x$ to $y$. Then,

$$
\chi\left(\bigcup_{v \in V} A_{v}\right)=\sum_{i \in V} \chi\left(A_{i}\right)-\sum_{\{i, j\} \in E} \chi\left(A_{i} \cap A_{j}\right) .
$$

Proof. Again, we apply Theorem 1. As in [6] we define for any non-empty subset $X$ of $V$,

$$
X^{*}:=\bigcup_{x, y \in X}\{z \in V \mid z \text { is on the path from } x \text { to } y\} .
$$

Then, each closed subset $X$ of $V$ has a unique base, namely the set of its leaves if $X$ is not a singleton, otherwise $X$ is a base of itself. It is easy to see that $X$ is perfectly closed if and only if $X$ is an edge or a singleton. Condition (7) is satisfied since any non-closed set $X \neq \emptyset$ contains $x$ and $y$ such that $A_{x} \cap A_{y} \subseteq A_{z}$ for some $z \notin X$ which lies on the path between $x$ and $y$. Thus, the corollary follows from Theorem 1.

The dual version of the following corollary has been published in [5] together with a generalization to Möbius inversion over power set lattices. Note that the dual version is obtained by replacing $v>\max C$ with $v<\min C$. As noted in [5], the result generalizes Whitney's famous broken circuit theorem [18].

Corollary 3 (Dohmen) Let $\left\{A_{v}\right\}_{v \in V}$ be a finite family of sets, where $V$ is endowed with a linear ordering relation, and let $\mathrm{C}$ be a set of non-empty subsets of $V$ such that for any $C \in \mathcal{C}$,

$$
\bigcap_{c \in C} A_{c} \subseteq A_{v} \text { for some } v>\max C .
$$


Then,

$$
\chi\left(\bigcup_{v \in V} A_{v}\right)=\sum_{\substack{J \in \mathcal{P}(V) \\ J \nsupseteq C(\forall C \in \mathcal{C})}}(-1)^{|J|-1} \chi\left(\bigcap_{j \in J} A_{j}\right) .
$$

Proof. For any non-empty subset $X$ of $V$ define

$$
X^{*}:=X \cup\left\{v \in V \mid \bigcap_{c \in C} A_{c} \subseteq A_{v} \text { and } v>\max C \text { for some } C \in \mathcal{C}, C \subseteq X\right\} .
$$

Then, each closed set $X$ has a unique base, namely

$$
X \backslash\left\{v \in V \mid \bigcap_{c \in C} A_{c} \subseteq A_{v} \text { and } v>\max C \text { for some } C \in \mathcal{C}, C \subseteq X\right\} .
$$

Clearly, $X$ is perfectly closed if and only if $X \nsupseteq C$ for any $C \in \mathcal{C}$. To verify (7) observe that for any non-closed $X \neq \emptyset$ there is some $C \subseteq X$ and $v \notin X$ such that $\bigcap_{c \in C} A_{c} \subseteq A_{v}$ and $v>\max C$; in particular, $\bigcap_{x \in X} A_{x} \subseteq A_{v}$ for some $v \notin X$. Thus, the requirements of Theorem 1 hold and the result follows.

Remark. Based on the recent theory of abstract tubes due to Naiman and Wynn [12,13], the author [3] proved that truncating the identity in Theorem 1 at some level $k$ gives a lower resp. upper bound for $\chi\left(\bigcup_{v \in V} A_{v}\right)$ depending on whether $k$ is even or odd. These new truncation bounds are provably at least as sharp as the classical Bonferroni bounds although less computational effort is required to compute them.

\section{Acknowledgement}

The author wishes to thank the referees for their valuable comments, in particular for simplifying the proofs of Proposition 1 and Proposition 2.

\section{References}

[1] K. Dohmen, An improvement of the inclusion-exclusion principle, Arch. Math. 72 (1999), 298-303.

[2] K. Dohmen, Improved inclusion-exclusion identities and inequalities based on a particular class of abstract tubes, Electron. J. Probab. 4 (1999), \# 5.

[3] K. Dohmen, Convex geometric inclusion-exclusion identities and Bonferroni inequalities with applications to system reliability analysis and reliability covering problems, Informatik-Bericht Nr. 132, Humboldt-Universität zu Berlin, 1999.

[4] K. Dohmen, Inclusion-exclusion and network reliability, Electron. J. Comb. 5 (1998), \# R36.

[5] K. Dohmen, A note on Möbius inversion over power set lattices, Commentat. Math. Univ. Carol. 38 (1997), 121-124.

[6] P.H. Edelman and R. Jamison, The theory of convex geometries, Geom. Dedicata 19 (1985), 247270. 
[7] H. Edelsbrunner, The union of balls and its dual shape, Discrete Comput. Geom. 13 (1995), 415440.

[8] H. Edelsbrunner and E.A. Ramos, Inclusion-Exclusion complexes for pseudodisk collections, Discrete Comput. Geom. 17 (1997), 287-306.

[9] J. Galambos and I. Simonelli, Bonferroni-type Inequalities with Applications, Springer-Verlag, New York, 1996.

[10] T.A. McKee, Graph structure for inclusion-exclusion equalities, Congr. Numer. 133 (1998), 121126.

[11] T.A. McKee, Graph structure for inclusion-exclusion inequalities, Congr. Numer. 125 (1997), 5-10.

[12] D.Q. Naiman and H.P. Wynn, Abstract tubes, improved inclusion-exclusion identities and inequalities and importance sampling, Ann. Statist. 25 (1997), 1954-1983.

[13] D.Q. Naiman and H.P. Wynn, Inclusion-exclusion-Bonferroni identities and inequalities for discrete tube-like problems via Euler characteristics, Ann. Statist. 20 (1992), 43-76.

[14] H. Narushima, Principle of inclusion-exclusion on partially ordered sets, Discrete Math. 42 (1982), 243-250.

[15] H. Narushima, Principle of inclusion-exclusion on semilattices, J. Combinat. Theory Ser. A 17 (1974), 196-203.

[16] G.C. Rota, On the foundations of combinatorial theory I. Theory of Möbius functions, Z. Wahrscheinlichkeitstheorie Verw. Geb. 2 (1964), 340-368.

[17] D.R. Shier, Network Reliability and Algebraic Structures, Clarendon Press, Oxford, 1991.

[18] H. Whitney, A logical expansion in mathematics, Bull. Amer. Math. Soc. 38 (1932), 572-579. 